\title{
PLCs AND SENSORS FOR CONTROLLING ELECTRO-PNEUMATIC POSITIONING SYSTEMS
}

\author{
Michail Papoutsidakis \\ Dept. of Industrial Design and \\ Production Engineering, \\ University of West Attica \\ Athens, Greece
}

\author{
V.J. De Negri \\ Dept. of Mechanical \\ Engineering, \\ Federal University of \\ Santa Catarina \\ Brazil
}

\author{
Efstathios Theocharis \\ Dept. of Industrial Design and \\ Production Engineering, \\ University of West Attica \\ Athens, Greece
}

\begin{abstract}
This project deals with automation in electropneumatic systems. Electro-pneumatics systems belong to the category automation systems and use electricity and compressed air for power. In the first chapter is made an import for pneumatics systems and we learn the historical development of them. In the second chapter we analyze the structure and the parts of the electro-pneumatic systems. There are compressors for compress the air, the pneumatics cylinders and valves and more kinds of electro-pneumatic systems. The third chapter analyzes all of the kinds of automation we have in electro pneumatic systems. In the beginning we see the pneumatic control, Hall, Reed, L.V.D.T and more sensors and then the PLC systems and how they work. Finally in the fourth chapter there are the conclusions of this project and the advantages of the electro-pneumatic systems.
\end{abstract}

\section{Keywords - Watermarking, Haar Wavelet, DWT, PSNR}

\section{INTRODUCTION}

\section{A. Introduction in Pneumatic Systems}

From ancient times to the nineteenth century, the systems used by humans were purely mechanical, with the help of levers and gears being controlled. With the evolution of technology and the above-mentioned requirements, other automation systems evolved.

Today in industry we use four types of automation systems: pneumatic, plumbing, electrical and electronic automation. Often two or more types are used, depending on the needs.

The pneumatic and hydraulic systems are mainly found in mechanical systems that need enough force and torque to activate them. In the industry, their main applications are robots, lifting devices and production lines. Implementation of pneumatic and hydraulic systems can also be found in cabs and aircrafts (hydraulic brakes, hydraulic clutches, active suspension, tilting rudders, movable surfaces of blades, pneumatic brakes)
The reason we encounter hydraulic and pneumatic systems in many applications is the ability to accumulate energy and then give it. One of the basic principles of the operation of these systems is to convert the flow to force and pressure, which will then be used to generate motion.

Hydraulic systems exploit the properties of liquids, which have very little compressibility, and use hydraulic fluids to transmit forces and movements. According to Pascal's law, each fluid transfers with unimpeded intensity the pressure it will receive on one surface of each other. They can develop great strengths and strengths for this and are suitable for industry applications that need to be exercised by great forces. They can work on high perspiration. Often, we will encounter mixed-type hydraulic systems that have the subsystem role in applications that need power transmission.

The pneumatic systems are driving automation systems. They have as an energy medium the compressed air and we use them for reciprocating movement of one or more pistons as well as more rarely for the rotation of motors. They operate at lower pressures than hydraulic systems and can not develop large forces but have lower costs and faster speeds of action. In industry, we often find such systems.

Electrical systems are the most widespread automation systems, with the use of electrical signals causing movements in other outputs. They need a lot of overloading precautions, but they are reliable and capable of moving away from complex systems.

Electronic systems also use electrical signals but much lower intensity, they have a very fast response time, they do not need much volume for their operation, which is several times a problem with the use of other systems, they have the lowest cost but the power transfer is limited and do not carry any forces at all. They are the only systems that are used in intelligent automation nowadays. 


\section{International Journal of Engineering Applied Sciences and Technology, 2019 Vol. 4, Issue 5, ISSN No. 2455-2143, Pages 417-424 \\ Published Online September 2019 in IJEAST (http://www.ijeast.com)}

\section{B. Historical review}

The name of the spiritual systems was given by the word "spirit" which in ancient Greek means wind, wind. Since ancient times, people have been using compressed air as a means of transporting energy. A typical example is the maintenance of flame with a bellows in a furnace that used it most for the processing of metals. Around 4000 BC probably in Mesopotamia used sails as a means of propulsion for their rafting vessels. Much later in the 1st century AD Herron of Alexandria, who is considered the first engineer to use compressed air in mechanical wheel drive devices, designed the windmill and left rich material for other constructions using pneumatic systems. The windmill had a horizontal axis of rotation and four fins.

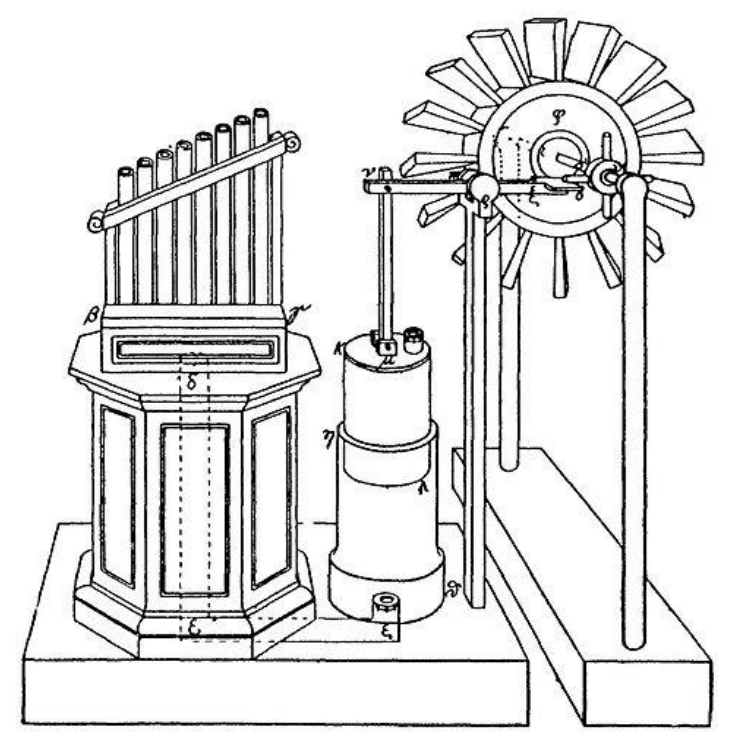

Fig. 1. Windmill of Heron 1st Century AD

More recently, in 1650, the German scientist Otto von Guericke invented the first vacuum pump, which consisted of a piston and a cylinder that could remove the air through containers. In 1654 he conducted an experiment with which he demonstrated the existence of atmospheric pressure. He placed 2 hollow hemispheres in such a way as to form a ball and with a leather ring ensure that the joint will be airtight and with the help of the vacuum pump remove the air between them. Then he tied each hemisphere to a group of 8 horses that could not leave the two hemispheres.

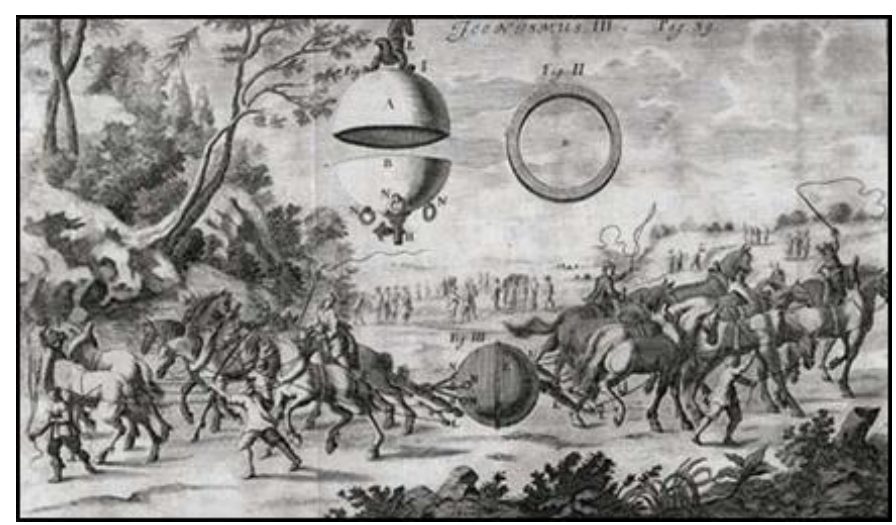

Fig. 2. Otto Von Guericke's First Vacuum Pump

In 1871 the pneumatic drill was invented which is the first spiritual tool. At the very next time, water-cooled air compressors appeared. From the 20th century to the present day, people have discovered many air-operated devices and a wide variety of automation systems using pneumatic systems.

\section{STRUCTURE AND PARTS OF ELECTRO-PNEUMATICS}

\section{A. General}

Automations that use compressed air for energy medium and result in reciprocating or rotating an engine are called pneumatic automation systems. They solve many automation problems in an easy and economical way and that's why they are widely spread these days.

The characteristics of pneumatic systems are:

- They can easily store and transport energy

- It is easy to adjust their speed and strength

- Produces low-cost, high-speed motion (1-2 m / sec) with a maximum distance of $2 \mathrm{~m}$ and a maximum force of $3000 \mathrm{Kp}$.

\section{B. Air Compressors}

For the operation of pneumatic systems, the air needs the pressure required for the operation of the system. This role is taken over by air compressors.

Air compressors produce compressed air. There are three main categories of air compressors, piston, rotary and flow compressors. In the piston compressors the air is introduced into a chamber and then the volume of the chamber is reduced, resulting in the air compressing. As far as rotary compressors are concerned, usually by means of a impeller, the kinetic energy is converted to static pressure. Finally, in air flow compressors the air is compressed by accelerating its mass.

Nowadays we use more rotary and piston compressors. The air entering it should be chilly, dry and free from dust. It is very crucial for their operation. 


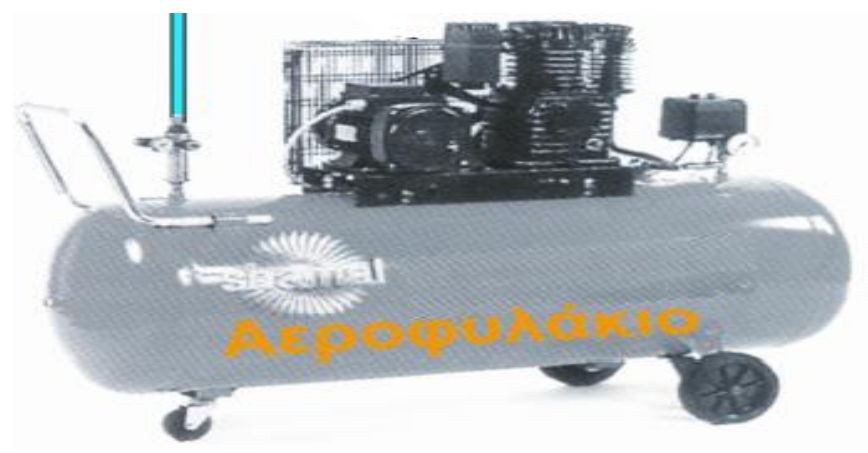

Fig. 3. Compressed Air Compartment

The compressed air then through pipelines, usually made of iron or copper, goes into the air chamber where it is stored. The pipelines are cylindrical in cross-section and are suitable to be tilted downwards in the order of $1-2 \%$ starting from the compressor compartment so that the moisture inside the system does not go to consumption and is driven towards lower point of the grid where a drainage valve is installed. Also the horizontal branches are of the ' $\mathrm{H}$ ' format. It is important that these specifications are complied with to ensure that the system is reliable and efficient for its better performance. Indicative is that for a pressure drop of $1 \mathrm{bar}$, the air compressor will need $7 \%$ more current.

The balloon or otherwise accumulator connected in series with the compressor is the compressed air storage for the grid. It is usually cylindrical in shape, made of steel and because of its large surface it facilitates cooling of the air and thus a percentage of the moisture will be removed and a smoothing of the pressure fluctuations in the grid is achieved.

The air in the grid can contain impurities that have either entered the compressor when air is sucked in or from the pipes used to transport the air. There may also be a problem, as we have said, with moisture or excessive lubrication. These elements would cause problems in the system's equipment, mainly disturbances, but also damage to its components.

This work has been carried out by the pressure regulator, the filter and the lubricator, subsystems that must include every typical mental system. The pressure regulator is required to keep the system operating pressure constant, regardless of the consumption. The purpose of the filter is to remove any dirt inherent in compressed air as well as water passing through the grid. The lubricator is required to feed the pneumatic system with a lubricant to keep the friction at low levels, and to reduce the wear and tear of the moving means of the system. Also the lubricator is the one that protects the elements of the system from corrosion.

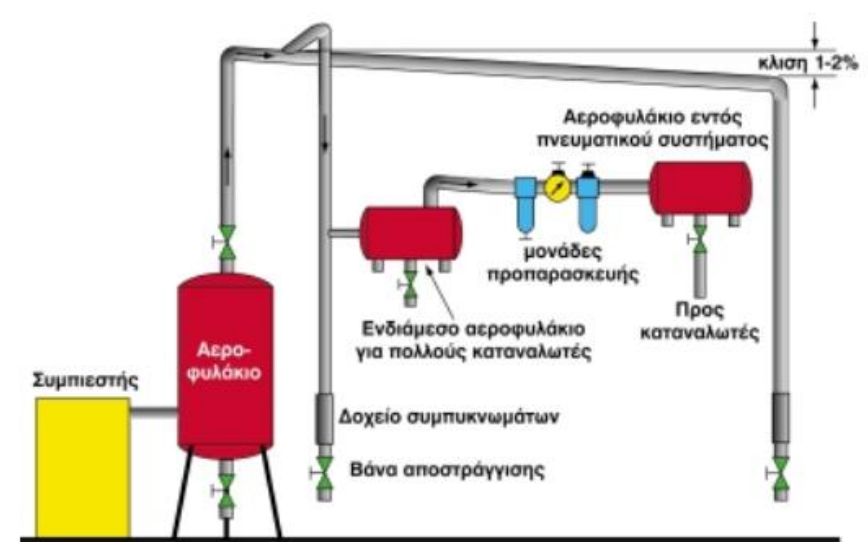

Fig. 4. Compressed Air Distribution Network

\section{Pneumatic Cylinders}

Many industrial applications require linear motion during their operating sequence. One of the simplest and most cost effective ways to accomplish this is with a pneumatic actuator, often referred to as an air cylinder. An actuator is a device that translates a source of static power into useful output motion. It can also be used to apply a force. Actuators are typically mechanical devices that take energy and convert it into some kind of motion. That motion can be in any form, such as blocking, clamping, or ejecting.

Pneumatic actuators are mechanical devices that use compressed air acting on a piston inside a cylinder to move a load along a linear path. Unlike their hydraulic alternatives, the operating fluid in a pneumatic actuator is simply air, so leakage doesn't drip and contaminate surrounding areas.

There are many styles of pneumatic actuators including diaphragm cylinders, rodless cylinders, telescoping cylinders and through-rod cylinders.

The most popular style of pneumatic actuator consists of a piston and rod moving inside a closed cylinder. This actuator style can be sub-divided into two types based on the operating principle: single acting and double acting.

Single-acting cylinders use one air port to allow compressed air to enter the cylinder to move the piston to the desired position, as well as an internal spring to return the piston to the "home" position when the air pressure is removed.

Double-acting cylinders have an air port at each end and move the piston forward and back by alternating the port that receives the high pressure air.

$\mathrm{n}$ a typical application, the actuator body is connected to a support frame and the end of the rod is connected to a machine element that is to be moved. An on-off control valve is used to direct compressed air into the extended port while opening the Retract port to atmosphere. The difference in pressure on the two sides of the piston results in a force equal to the pressure differential multiplied by the surface area of the piston.

If the load connected to the rod is less than the resultant force, the piston and rod will extend and move the machine element. 


\section{International Journal of Engineering Applied Sciences and Technology, 2019 \\ Vol. 4, Issue 5, ISSN No. 2455-2143, Pages 417-424 \\ Published Online September 2019 in IJEAST (http://www.ijeast.com)}

Reversing the valving and the compressed air flow will cause the assembly to retract back to the "home" position.

Pneumatic actuators are at the working end of a fluid power system. Upstream of these units, which produce the visible work of moving a load, are compressors, filters, pressure regulators, lubricators, on-off control valves and flow controls. Connecting all of these components together is a network of piping or tubing (either rigid or flexible), and fittings.

Pressure and flow requirements of the actuators in a system must be taken into account when selecting these upstream system components to ensure desired performance. Undersized upstream components can cause a pneumatic actuator to perform poorly, or even make it unable to move its load at all.

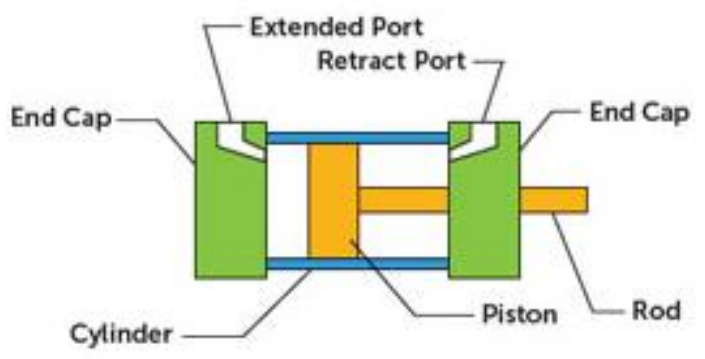

Fig. 5. Cylinder Double Acting

\section{Valves}

The elements that make up a spiritual system can be classified into the following categories:

- Signal delivery and processing

- Air supply signal valves

- Signal processing valves (pressure regulator, timing valve)

- Centrifugal directional valves

- Air pistols that provide airflow to the system

The treatment can also be done between the control valve and the piston, not necessarily just between the signal valve and the control valve.

Beyond the piston which is the most important element together with the air of a system it is necessary to use a control valve for each air piston separately. Put it as close as possible to the air piston. For activation it needs to reach a signal or several times and two or more signals that come from the signal valves where it can be either start or stop. Generally, when there is more than one piston, each is followed by its own control valve, signal valve and processing valve so that each is a subsystem of the main pneumatic system

Between the piston and control valve, regulating valves are inserted for the automation systems that process the airflow to the pistons. Such are the flow control valves and the nonreturn valves.

Also very important is that both the design of the system for its construction and the final design of the system is always in a state of calm, that is, it has not started any function.
Valves are devices that help us either to adjust the start or stop of a piston or to determine the direction of flow of compressed air. Their name is defined as a basis for international terminology and symbols are used to display symbols according to DIN ISO 1219. Thus, the valves according to their function are divided into the following categories:

- Check valves

- Signal valves

- Regulating valves

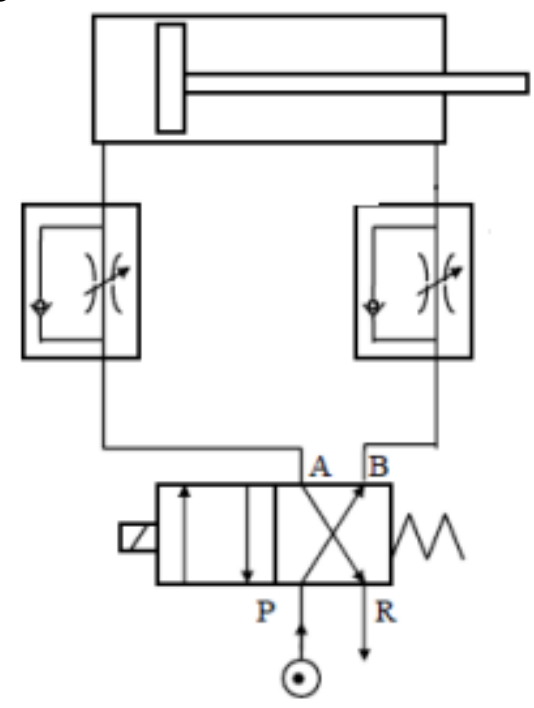

Fig. 6. Cylinder Double Acting with Two Valves

\section{AutOMATION IN PNEUMATICS}

\section{A. Programmable logic controllers}

\section{A. General}

With the revolution of computer science, the production of microcomputers began. In industry, most of the automations were used by relays automation, and in the early 1970s PLCs would appear to simplify the circuits and replace the many relays, timers, timers, logic logic boards, auxiliary contacts of relays and counters. Indeed, when they appeared in the industry, their full name was not known, which is Programmable Logic Controller, that is, programmable logic controllers not to scare the technical establishment of the industry.

The PLC is a microcomputer suitably constructed to be used for automation operations. It was created to replace the classic multi-relay table and to integrate a piece of programming into the electronic systems and to combine them with other types of automation to solve the applications. Because this transition would not be easy, the first programming languages for the PLCs were simplistic and what they did basically was to copy the electrical design to a specific programming device. Nowadays PLCs have evolved rapidly and there are many different models in the market. 


\section{International Journal of Engineering Applied Sciences and Technology, 2019 Vol. 4, Issue 5, ISSN No. 2455-2143, Pages 417-424 \\ Published Online September 2019 in IJEAST (http://www.ijeast.com)}

More specifically, PLCs are digital electronic devices with programmable memory in which commands are stored which can perform logical, sequential, time and numerical functions. Today with microprocessor technology and modern software in the foreground, the features and capabilities of programmable logic controllers have changed to an extent that influenced the design philosophy of control systems. Thus, the current PLCs have the ability to connect to local networks, collect information directly from any sensor, and perform analog control algorithms.

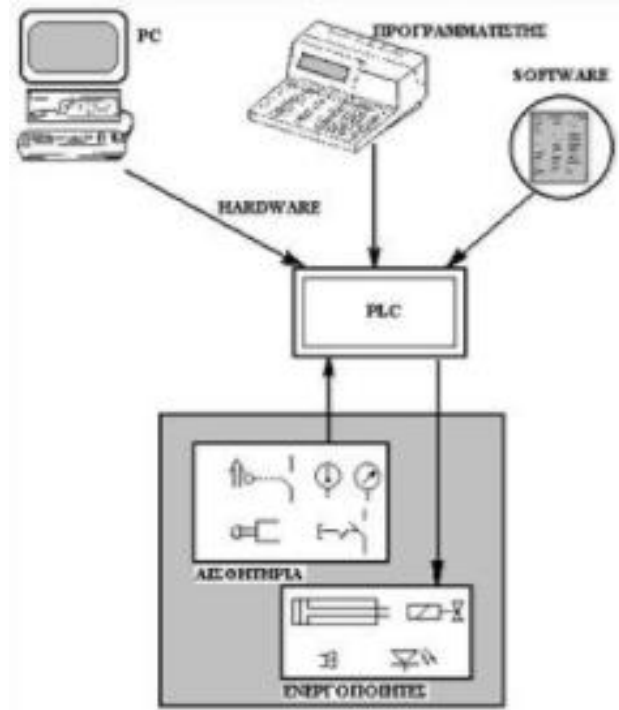

Fig. 7. Control System Using PLC

The PLCs consist of the Central Processing Unit (CPU), which is also its master, power supply unit and input-output modules. All of this is the main part of a programmable logic controller and most models manufacture these three devices built into one. Apart from the main part, a PLC is made up of the frame for mounting the modules and their extensions and the programming device with which the PLC is programmed. They also have RAM, EEPROM, ROM and special functions such as timers, counters, comparators, pulse generators, and real-time counter.

PLCs are programmed in three languages:

- In LADDER, which is the first language to develop and allows the transmission of the electrical design via the programming device.

- In STL which is the logical command language. This programming language contains commands that correspond to logical gates. Nowadays it has been enriched with elements of the Assembly language.

- In logical graphics language (FBD). In this programming language, instead of the electrical plan, the corresponding logic circuit is used.
The PLCs we mentioned were an electronic layout that could be simulated by an automation panel from a functionality point of view. It has inputs and outputs associated with the components of an installation that are specified for what they will do from an algorithm. This algorithm is responsible for the outputs of the system according to the signals it has from the inputs. An example might be that an engine shuts off when a switch is activated.

The special feature of PLCs is that the movements that will be made in the automation system are not fixed as in an automation panel but can be altered by the operation in the program that runs without changing anything in the system circuit. The PLC CPU contains the automation logic that reads the state of the inputs at any moment if they are broken or not and according to the program stored in its memory it activates the appropriate outputs. In addition to the appropriate output, it arms a relay that can trigger a motion device or open some lights through it.

\section{B. PLC Mode}

In practice, the CPU initially reads all the system inputs and checks each one separately if a voltage is displayed, and if it displays, it registers a logic 1 to a memory location built for that purpose. Thus, the state of the inputs is now contained in the memory. Then run the program based on which we will give the values at the system outputs that are also entered into special output image locations. Finally, the output memory locations are transferred to the output card and the following moves are made, such as the activation of a relay. This process is repeated continuously so that each change of status at the inputs is recognized and the system performs the corresponding activation of the hexes. This process is called detection cycle.

Most CPUs have a very short detection cycle of around $3 \mathrm{msec}$, but for highly critical inputs there are techniques that allow instant CPU information. Thus, during the detection cycle the information about the state of the input is obtained at its beginning and as long as the program is executed it is considered stable. Once the cycle is complete in these fractions of a second, it reads the state of the inputs again and will act on any changes that have occurred since the previous cycle. As we mentioned any change we want to do in the system, we do it by changing only the program and not the wiring of the system and that is what makes programmable logic controllers in the industry very useful.

In order to implement a PLC automation, some work steps must be followed. Initially we need to record system requirements that we want to create even the future extensions we may want to do, choose the type and number of PLCs we will use based on the best technical solution at the lowest cost. Next, the panel is built and then installed and connected to the peripherals and the necessary tests are performed for the 


\section{International Journal of Engineering Applied Sciences and Technology, 2019 \\ Vol. 4, Issue 5, ISSN No. 2455-2143, Pages 417-424 \\ Published Online September 2019 in IJEAST (http://www.ijeast.com)}

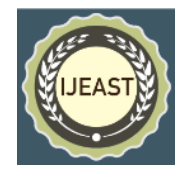

correct operation so that the program is finally transferred to the PLC and a project folder is created with all designs.

\section{Main Functions}

Nowadays, PLCs perform several functions that help create automation. These functions increase as the PLCs evolve at a very fast pace.

A very important element of PLCs is the counter, which can count internal or external pulses. The enumeration can be either up or down. A feature that is also important is the ability to program in real time, date and time the activation of some outputs and the time units that are the units of time measurement. Also, modern programmable logic controllers can process numerical operations.

As we have said, PLCs have come to replace wired logic automations and have evolved so that they can also fully cover auto-control systems. This is possible as PLCs are able to receive and process analogue inputs as well as provide analogue outputs. Convert analog input values to digital values and then process these values by utilizing the possibilities for digital number processing.

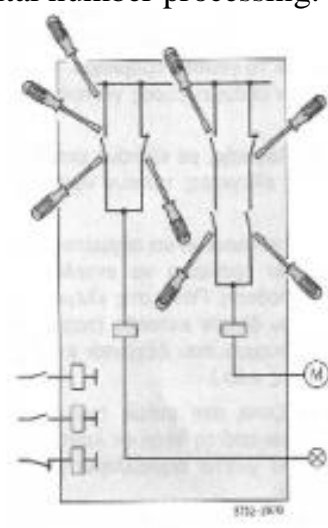

EYPMATOEH

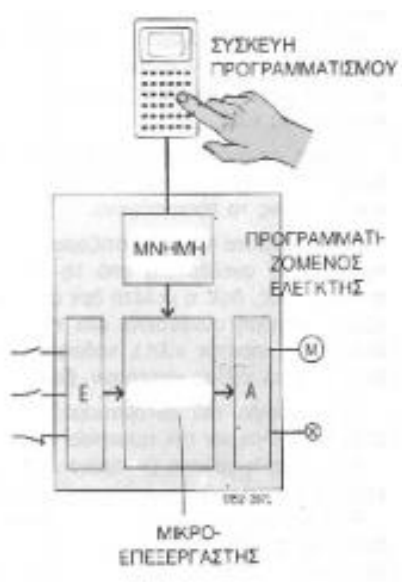

Fig. 8. With Wiring and Using PLC
Finally, PLCs can work together and with computers. With this function we can exchange information between systems and connect the programmable logic controllers with computers that are responsible for controlling all production and controlling the warehouse and the factory account. All of this together is a key industrial automation network.

\section{B. Pneumatic control}

\section{A. General}

In spiritual systems to place a mental control system, we follow a series of actions to get the best performance for our system, taking into account all the conditions and situations that may exist

Initially we should clearly define the goals we have set for this system, what we want to achieve. Also, some conditions for the system's operation, such as the startup, layout and security condition, and some operational influences such as installation space, environment, and system delivery must be defined.

As we have mentioned, it is often used 2 different automation systems. So when we study cost control and better technical choice for the control system we can have different types of automation systems. With the use of specific components, we can combine mechanical, electrical, pneumatic or hydraulic systems.

Also very important is the schematic depiction of the spiritual control we are examining. In this way, we can easily identify the parameters that relate to the work items as well as their working method and how they will be placed.

Finally, all movements of the intellectual system must be reproduced in a clear way so that it is easy to understand later on either for maintenance or to make it easy to repair any damage to the system. There are a number of ways to represent the system, but for each spiritual system it can be chosen differently to combine some of them according to the system we have. When designing, the system is always at rest.

\section{B. Pressure sensors}

The pressure as we have stated is defined as the force exerted on a surface through this surface. So to measure the pressure, it is sufficient for a known surface to measure its strength. Most of the time, sensors are used in the industry.

Devices for measuring and controlling pressure are divided into two basic types, the force comparing type and the type of elastic deformation. In the first category the pressure is measured by the force exerted on a known area of area. Manometers and weighing devices are used.

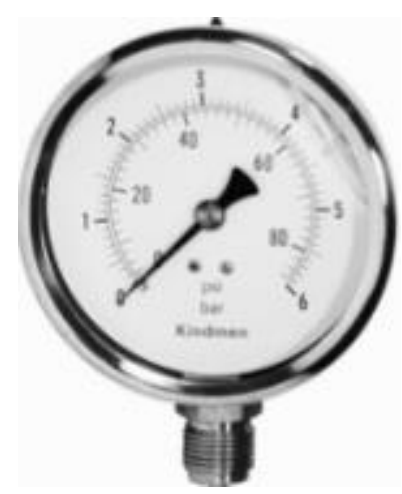

Fig. 9. Pressure Gauge 0-6 bar

In the type of elastic deformation, elastic mechanical elements are used which are deformed under the effect of the air pressure resulting in the actuation of an electrical switch which will also provide electrical information to the control system. In this category are the pressure switches. 


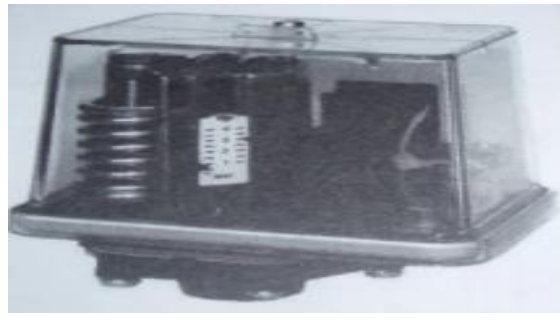

Fig. 10. Pressure Switch

\section{Position Sensors}

\section{A. Terminal switches}

Terminal switches are electrical devices that I use to get a signal in the control system when they come into contact with the objects they are scanning. They are very often used to detect the limits of a movement and are therefore called limit switches. The closed switches may be normally open.

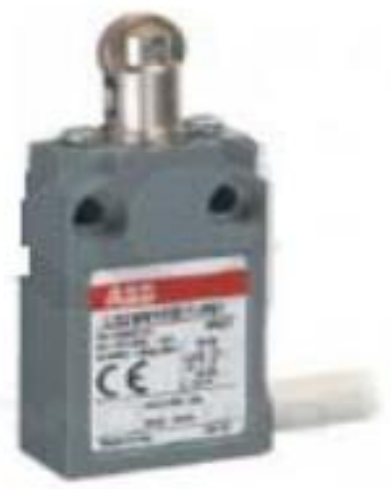

Fig. 11. Terminal Switch

Terminal switches are one of the most popular positioning sensors in the industry, but also in many automation systems. Because the range of applications and their needs is very large, there is a wide variety of kinds of terminal switches.

Various kinds of terminal switches consist of how to attach and configure the rotor. The cursor is the movable element of the switch on which the object will be touched to detect and the switch is closed. The task is, depending on the object of detection, that the cursor is the most appropriate to minimize the effect on the movement of the object and to ensure that the detection is reliable. Also in some systems where there are disturbances due to bounce signals when switched from on to off, damping circuits are used. The damping circuit capacitor of the bounce signals ground up the disturbances that occur, which, if analyzed in the frequency range, are very high.

\section{CONCLUSIONS}

As we have seen in detail the pneumatic systems are those that use as the energy medium the compressed air and result in the reciprocating movement of a piston or the rotation of an engine. The reasons that are used in many applications and that they are still evolving even nowadays are that they are initially easy to store but also transport, energy, there is no risk of explosion or overcharging and can operate under abnormal environmental conditions as well as do not pollute but are environmentally friendly. Also very important for every company-company, the cost of compressed air production is low as well as the creation of linear motion at high speeds and convinced air can be used anywhere in inexhaustible quantities. Finally, in the pneumatic systems an advantage we find is that we have the ability to easily adjust the speed and force of compressed air and that all the elements of the system are easy and economical to maintain and repair a fault

Electro-pneumatic systems are a combination of pneumatic and electrical systems. Using electro-pneumatic systems has several advantages. Transmission of signals takes place in a much faster time than in pneumatic systems, as the signals are electric. The energy required to send signals to electro pneumatic systems is much lower. We also have more control capabilities because we can convert physical sizes such as temperature, pressure or humidity into analogue electrical signals. Finally, electro pneumatic systems have more economical materials and their figures are of smaller magnitude than those of the pneumatic systems.

In the following work we mentioned the automation items in the electro-pneumatic systems using position sensors, terminals and microprocessors. As we have seen, PLCs are industry computers that receive input signals from switches and sensors. They then evaluate the input signals according to a stored program and generate signals to control either machines or generic manufacturing processes. The programmable logic controller was created to replace the classic multi-relay table, meter timers and also to integrate a piece of programming into electronic systems and to combine them with other types of automation to solve the applications. By using them the electric control circuitry of the machines is simplified on the substance it becomes smarter and more flexible in conversions as well as more reliable in operation. A large piece of control circuit wiring enters the PLC program.

Nowadays where productivity is a very important factor for every business to achieve its goals, the choice of automation systems to use is very decisive. PLCs have several advantages over conventional systems. Initially, their cost permits the simplest machine that performs an electrical control to use them, they have such capabilities that they can use in various applications and their programming is relatively easy. It is possible to change the process in the operation of a PLC- 
controlled machine by varying its program without changing anything in the wiring. Something that is very important and is probably the biggest advantage of PLCs. Finally, the productivity of machines with their use increases, automation becomes easier and less damage occurs.

\section{ACKNOWLEDGMENTS}

All authors would like to thank the University of West Attica for the financial support provided to them for this research project.

\section{REFERENCES}

[1] Hydraulic-Pneumatic Systems and Applications, Athanasios T. Routoulas, Synchronous Publishing, Athens 2008

[2] History of Technology and Automata, S. Vassiliadou and D. Kalligeropoulos, Modern Publishing, Athens, 2005.

[3] http://www.pronews.gr/istoria/177882_o-iron-oalexandreys-kai-i-viomihaniki-epanastasi-poy-argise2000-hronia-vinteo

[4] https://el.wikipedia.org/wiki/\%CE\%A0\%CE\%BB\%CE\% $\mathrm{BF} \% \mathrm{CE} \% \mathrm{AF} \% \mathrm{CE} \% \mathrm{BF}$

[5] https://el.wikipedia.org/wiki/\%CE\%8C\%CF\%84\%CE\%B F_\%CF\%86\%CE\%BF\%CE\%BD_\%CE\%93\%CE\%BA\% CE\%AD\%CF\%81\%CE\%B9\%CE\%BA\%CE\%B5

[6] http://ebooks.edu.gr/modules/ebook/show.php/DSGYMB200/530/3511,14401/

[7] http://edume.myds.me/00_0070_e_library/10030/03_Auto mation_Books/003/09.pdf

[8] https://repository.kallipos.gr/bitstream/11419/6262/5/00 master_document_VER_6-KOY.pdf

[9] http://nefeli.lib.teicrete.gr/browse/stef/mhx/2014/Xenikak isGeorgios/attached-document-1405403417-5211377668/Xenikakis_Georgios.2014.pdf

[10] https://apothesis.lib.teicrete.gr/bitstream/handle/11713/21 06/LoliMarianthi2013.pdf?sequence $=1$

[11] http://okeanis.lib.teipir.gr/xmlui/bitstream/handle/123456 789/3871/ntst8.pdf?sequence $=1$

[12] http://dspace.lib.ntua.gr/dspace2/bitstream/handle/123456 789/562/themelisn_plc.pdf?sequence $=1$

[13] http://robotics.mech.upatras.gr/files/theses/dimeas_studen t_project.pdf

[14] file:///C:/Users/User01/Downloads/Nimertis_Tsikouris(el e) $\% 20(2)$.pdf

[15] file:///C:/Users/User01/Downloads/marinosd_pump.pdf

[16] http://apothesis.teicm.gr/xmlui/bitstream/handle/1234567 89/673/kapsioxas.pdf?sequence=1\&isAllowed=y 